\title{
Gauge invariance of meson photo- and electroproduction currents revisited
}

\author{
Helmut Haberzettl@* \\ Institute for Nuclear Studies and Department of Physics, The George Washington University, \\ Washington, DC 20052, USA
}

(Received 25 May 2021; accepted 3 August 2021; published 2 September 2021)

\begin{abstract}
An exact expression is derived for the meson production current off the nucleon for real and virtual photons which cleanly separates, in a model-independent manner, a tree-level expression that manifestly preserves local gauge invariance in terms of a generalized Ward-Takahashi identity from transverse finalstate-interaction (FSI) terms. As discussed in some detail, this exact formulation is particularly well suited for implementing approximation schemes of exchange-current and FSI contributions (which in practice generally will be necessary) at any level of sophistication without violating local gauge invariance.
\end{abstract}

DOI: 10.1103/PhysRevD.104.056001

\section{INTRODUCTION}

Local gauge invariance-the requirement that physical observables are invariant under local U(1) transformations of fields-implies the very existence of the electromagnetic gauge field $A^{\mu}$ and thus it is one of the fundamental symmetries of electromagnetic physics [1]. Conversely, any violation of this symmetry in theoretical reaction models means that damage has been done to the underlying description of the electromagnetic field. For problems with complex reaction dynamics, in particular, where approximations generally are unavoidable, local gauge invariance is oftentimes lost and some effort must be made to repair this damage.

For pion photoproduction off the nucleon, the necessary and sufficient condition for local gauge invariance was established by Kazes [2] in terms of a generalized WardTakahashi identity for the four-divergence of the corresponding production current. This identity is easily verified for the tree-level description of the process for structureless particles without form factors, but it fails for more realistic descriptions with form factors. To our knowledge, the earliest suggestion how to remedy the situation was given by Drell and Lee [3] who proposed an additional nonsingular contact current as a way to summarily account phenomenologically for all higher-order contributions (like final-state interactions etc.) neglected at the tree level. Gross and Riska [4] presented a repair scheme for gauge invariance that reinterprets vertex dressing effects as

\footnotetext{
*helmut.haberzettl@gwu.edu
}

Published by the American Physical Society under the terms of the Creative Commons Attribution 4.0 International license. Further distribution of this work must maintain attribution to the author(s) and the published article's title, journal citation, and DOI. Funded by SCOAP. self-energy contributions for propagators connected to the vertex and modifies electromagnetic single-particle currents such that this self-energy change is reflected in their Ward-Takahashi identities. The ad hoc Drell-Lee recipe was later rediscovered independently by Ohta [5] in a constructive procedure implementing the electromagnetic interaction at the pion-nucleon vertex utilizing expansions and making use of the minimal-substitution rule. In these gauge-invariance-fixing schemes kinematic singularities are avoided for the contact current by constructing suitable nonsingular $0 / 0$ expressions whose fourdivergences cancel gauge-invariance-violating terms. A seemingly different method proposed by Nagorny and Dieperink [6] in terms of a contact current written as a nonsingular interpolating integral can in fact be shown to be functionally equivalent to the Drell-Lee-Ohta procedure. A generalization of the latter approach was put foward by Haberzettl $[7,8]$ within a consistent field-theory framework for describing electromagnetic meson production off baryons, including exchange currents and hadronic final-state interactions (FSI), with mesons and baryons as the relevant degrees of freedom. This framework also suggested a flexible method for maintaining gauge invariance in a manifest manner, even when approximating mechanisms like FSI or incorporating Regge exchanges [9-12]. The generalization also avoids the "violation of scaling behavior" [3] of the original Drell-Lee-Ohta recipe at high energies.

In all of these approaches the problem of maintaining gauge invariance and approximating higher-order dynamical mechanisms are intricately linked. In the present work, we will revisit the question of preserving local gauge invariance even for problems with rich internal dynamics that generally require approximations in practice by cleanly separating the issue of gauge invariance from approximations of reaction dynamics. We will provide a novel formulation of the production current that separates out, 
in a model-independent, exact manner, manifestly gaugeinvariance-preserving (GIP) tree-level expressions from transverse final-state-interaction (FSI) terms. This formulation thus allows devising efficient approximations to account for microscopic reaction mechanisms like exchange-current contributions and final-state interactions independent of gauge invariance. Local gauge invariance will always be preserved and never be at issue.

In Sec. II, we will briefly recapitulate expressions for the production current relevant for the present approach. Section III will then address local gauge invariance and provide the desired reformulation, including a discussion of possible approximations that all maintain manifest gauge invariance. The final Sec. IV provides a summarizing assessment.

\section{PRODUCTION CURRENT}

Taking nucleons $(N)$ and pions $(\pi)$ as generic placeholders for baryons and mesons, the meson-production process

$$
\gamma(k)+N(p) \rightarrow \pi(q)+N\left(p^{\prime}\right)
$$

for real or virtual photons, is described by a production current

$$
M^{\mu}=M^{\mu}\left(q, p^{\prime} ; k, p\right)
$$

that may be decomposed as [7]

$$
M^{\mu}=M_{s}^{\mu}+B^{\mu}+X G_{0} B^{\mu},
$$

represented by the diagrams of Fig. 1(a). Here, $M_{s}^{\mu}$ is the $s$-channel production current and $B^{\mu}$ is the 2-particleirreducible Born mechanism,

$$
B^{\mu}=M_{u}^{\mu}+M_{t}^{\mu}+M_{c}^{\mu},
$$

depicted in Fig. 1(b), that contains the usual $u$ - and $t$-channel contributions and the interaction current

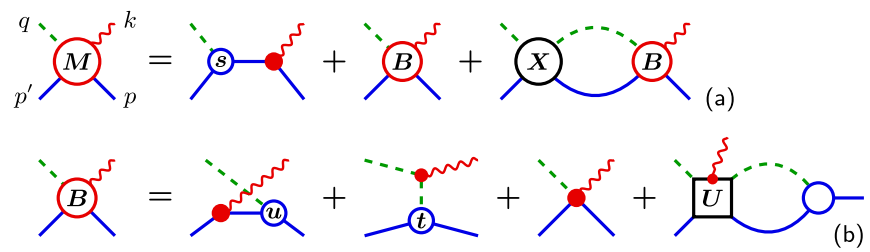

FIG. 1. (a) Production current $M^{\mu}$ of Eq. (3). (b) Born-type mechanisms $B^{\mu}$ of Eq. (4). The last two diagrams provide the interaction current $M_{c}^{\mu}$ of Eq. (5). Labels $s, u$, and $t$ refer to Mandelstam variables for the respective kinematic situations of hadronic vertices. Time proceeds from right to left to maintain one-to-one correspondence between equations and diagrams.

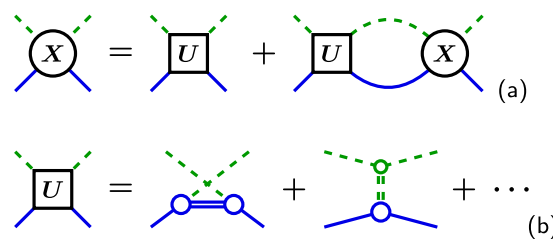

FIG. 2. (a) Integral equation for the hadronic meson-baryon scattering amplitude $X$ of Eq. (6), with (b) nonpolar driving terms $U$; ellipses indicate higher-order loop contributions [7]. Solid and dashed double lines subsume summations over all possible intermediate baryons and mesons compatible with external meson-baryon states.

$$
M_{c}^{\mu}=m_{c}^{\mu}+U^{\mu} G_{0} F
$$

given by a Kroll-Ruderman-type elementary contact current $m_{c}^{\mu}$ (which may be absent depending on the underlying hadronic coupling scheme) and a loop integration over the pion-nucleon vertex $F$ and exchange currents $U^{\mu}$. The finalstate interaction $X$ for meson-nucleon scattering is determined by the integral equation

$$
X=U+U G_{0} X,
$$

shown in Fig. 2(a), driven by the 2-particle-irreducible exchanges $U$ of Fig. 2(b). (The exchange currents $U^{\mu}$ occurring in Eq. (5) are given by attaching a photon to this driving term $U$, as depicted in Fig. 3.) The amplitude $X$ is not the full physical meson-nucleon scattering amplitude $T$; the latter is obtained by adding a fully dressed $s$-channel pole term to $X$ [7]. ${ }^{1}$ The intermediate free meson-nucleon Green's function is given by

$$
G_{0}=t_{\pi}\left(q_{\pi}\right) \circ S\left(P-q_{\pi}\right)
$$

where $t_{\pi}$ describes the meson propagator with four-momentum $q_{\pi}$ and $S$ provides the nucleon propagator depending on the remaining four-momentum $P-q_{\pi}$, where $P=k+$ $p=q+p^{\prime}$ is the total four-momentum of the system. The symbol " $\circ$ " stands for the convolution integral over the loop momentum $q_{\pi}$. Recalling that the terms pion and nucleon (and the respective indices $\pi$ and $N$ ) are just representative placeholders for all possible mesons and baryons, Eq. (3) is to be read as a system of equations for all intermediate meson-baryon states compatible with the external states, with all summation indices suppressed. As written, therefore, the form of Eqs. (3) and (6) is deceptively simple to provide notational clarity. In reality, this is a highly

\footnotetext{
${ }^{1}$ In general, a splitting into pole and nonpole terms is not unique, however, consistency requirements of the field-theory approach of Ref. [7] make it well defined here. Note in this context that while it is possible to write the FSI for the production current directly in terms of $T$, this will be at the expense of a very complicated, unusual $s$-channel contribution $[7,10]$.
} 


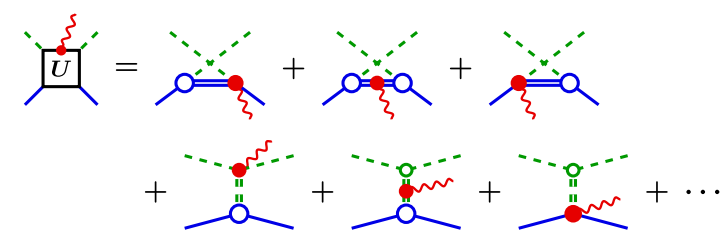

FIG. 3. Exchange currents $U^{\mu}$ obtained by coupling photons to the vertices and propagators of the driving term $U$ of Fig. 2(b).

complex coupled-channel problem that generally cannot be solved exactly without efficient approximation schemes.

\section{LOCAL GAUGE INVARIANCE}

Assuming that the description of the production current $M^{\mu}$ as given is complete at the level of meson and baryon degrees of freedom, the necessary and sufficient condition for its local gauge invariance is the generalized WardTakahashi identity (gWTI) [2,7],

$$
k_{\mu} M^{\mu}=-F_{s} S \hat{Q}_{N} S^{-1}+S^{-1} \hat{Q}_{N} S F_{u}+t_{\pi}^{-1} \hat{Q}_{\pi} t_{\pi} F_{t},
$$

where the functions $F_{x}$, for $x=s, u, t$, describe the pionnucleon vertices $F$ in $s-, u$, and $t$-channel kinematics,

$$
\begin{aligned}
F_{s} & \equiv F\left(q, p^{\prime} ; p+k\right), \\
F_{u} & \equiv F\left(q, p^{\prime}-k ; p\right), \\
F_{t} & \equiv F\left(q-k, p^{\prime} ; p\right),
\end{aligned}
$$

including hadronic coupling operators and isospin structures, with the order of momentum arguments given as outgoing pion and nucleon momenta on the left of the semicolon and incoming nucleon momentum on the right. In the notation here, the operators with hats, $\hat{Q}_{N}$ and $\hat{Q}_{\pi}$, indicate the charge operators $Q_{N}$ and $Q_{\pi}$ for the nucleon and pion, respectively, that also inject the incoming photon four-momentum $k$ for the particle they pertain to into the equation at their respective locations. With external fourmomenta given as in Eq. (1), this means that all momenta can be recovered easily and unambiguously and do not need to be spelled out explicitly, thus providing, for example,

$F_{s} S \hat{Q}_{N} S^{-1} \equiv F\left(q, p^{\prime} ; p+k\right) S(p+k) Q_{N} S^{-1}(p)$,

and similar expressions for $u$ - and $t$-channel terms. In other words, the charge operators $\hat{Q}_{n}(n=N, \pi)$ as used here cannot be moved through the expressions; they must remain at the locations where written. The inverse propagators appearing in the gWTI (8) imply that $k_{\mu} M^{\mu}=0$ for external on-shell hadrons thus providing current conservation. In general, however, Eq. (8) holds true irrespective of whether hadrons are on-shell and whether the photon is real or virtual.
Using the Ward-Takahashi identities [13,14] for the electromagnetic single-particle currents of nucleons $\left(J_{N}^{\mu}\right)$ and pions $\left(J_{\pi}^{\mu}\right)$,

$$
\begin{gathered}
k_{\mu} J_{N}^{\mu}=S^{-1} \hat{Q}_{N}-\hat{Q}_{N} S^{-1}, \\
k_{\mu} J_{\pi}^{\mu}=t_{\pi}^{-1} \hat{Q}_{\pi}-\hat{Q}_{\pi} t_{\pi}^{-1},
\end{gathered}
$$

and defining a longitudinal current

$$
C_{\mathrm{L}}^{\mu}=\frac{k^{\mu}}{k^{2}}\left(-F_{s} \hat{Q}_{N}+\hat{Q}_{N} F_{u}+\hat{Q}_{\pi} F_{t}\right),
$$

the four-divergence of this current and of the $s^{-}, u^{-}$, and $t$-channel Born terms produces

$$
\begin{aligned}
& k_{\mu}\left(M_{s}^{\mu}+M_{u}^{\mu}+M_{t}^{\mu}+C_{\mathrm{L}}^{\mu}\right) \\
& \quad=-F_{s} S \hat{Q}_{N} S^{-1}+S^{-1} \hat{Q}_{N} S F_{u}+t_{\pi}^{-1} \hat{Q}_{\pi} t_{\pi} F_{t},
\end{aligned}
$$

equal to the right-hand side of the gWTI (8). Hence, rewriting the production current (3) equivalently as

$$
\begin{aligned}
M^{\mu}= & \overbrace{M_{s}^{\mu}+M_{u}^{\mu}+M_{t}^{\mu}+C_{\mathrm{L}}^{\mu}}^{\mathrm{GIP}} \\
& +\left(M_{c}^{\mu}-C_{\mathrm{L}}^{\mu}\right)+X G_{0}\left(M_{u}^{\mu}+M_{t}^{\mu}+M_{c}^{\mu}\right),
\end{aligned}
$$

the terms labeled GIP already produce the gWTI. The remaining terms, therefore, must vanish when taking their four-divergence. Formally, we may then equate the current $C_{\mathrm{L}}^{\mu}$ of (12) with

$$
C_{\mathrm{L}}^{\mu}=\left[M_{c}^{\mu}+X G_{0}\left(M_{u}^{\mu}+M_{t}^{\mu}+M_{c}^{\mu}\right)\right]_{\mathrm{L}},
$$

where the bracket $[\cdots]_{L}$ denotes the longitudinal part of the enclosed current. The entire production current now reads equivalently

$$
\begin{aligned}
M^{\mu}= & M_{s}^{\mu}+M_{u}^{\mu}+M_{t}^{\mu}+C_{\mathrm{L}}^{\mu} \\
& +\left[M_{c}^{\mu}+X G_{0}\left(M_{u}^{\mu}+M_{t}^{\mu}+M_{c}^{\mu}\right)\right]_{\mathrm{T}},
\end{aligned}
$$

where $[\cdots]_{\mathrm{T}}$ indicates that only transverse parts of the bracketed current enter here, which do not contribute to the gWTI (8).

For practical purposes and to circumvent the technical issues associated with the $1 / k^{2}$ singularity in $C_{\mathrm{L}}^{\mu}$, we may rewrite Eq. (16) equivalently as

$$
\begin{aligned}
M^{\mu}= & M_{s}^{\mu}+M_{u}^{\mu}+M_{t}^{\mu}+C^{\mu} \\
& +\left[M_{c}^{\mu}-C^{\mu}+X G_{0}\left(M_{u}^{\mu}+M_{t}^{\mu}+M_{c}^{\mu}\right)\right]_{\mathrm{T}},
\end{aligned}
$$

where $C^{\mu}$ is the phenomenological, nonsingular gauge-invariance-preserving (GIP) current constructed in Refs. [7,9] which reproduces the necessary condition 


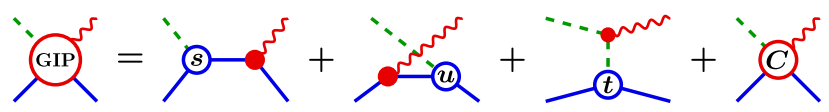

FIG. 4. Gauge-invariance-preserving tree-level part of Eq. (17), with the last diagram representing the contact-type GIP current $C^{\mu}$. These diagrams also correspond to the lowest-level approximation of the production current $M^{\mu}$ given in Eq. (21).

$$
k_{\mu} C^{\mu}=-F_{s} \hat{Q}_{N}+\hat{Q}_{N} F_{u}+\hat{Q}_{\pi} F_{t}
$$

to ensure the gWTI (8). The particular details of the construction in Refs. [7,9] are omitted here because they are not relevant for the present considerations. ${ }^{2}$ In fact, any (contact-type, nonsingular) current $C^{\mu}$ that satisfies (18) will imply the manifest preservation of local gauge invariance. The gauge-invariance-preserving tree-level part of Eq. (17) is depicted in Fig. 4. Because of the subtraction of $C^{\mu}$ within $[\cdots]_{\mathrm{T}}$, the exact equation (17) does not depend on the transverse part of $C^{\mu}$ which is the only part that is model dependent. The subtraction thus avoids double-counting of mechanisms already accounted for explicitly in $C^{\mu}$ outside of the $[\cdots]_{\mathrm{T}}$ bracket. This becomes particularly relevant when one considers approximations of $M_{c}^{\mu}$ or final-state interactions-which generally will be necessary in practically applications. Some straightforward approximation schemes are discussed in Sec. III A.

Equation (17) is the main result of the present paper. By construction, it provides an exact expression for the production current that is manifestly gauge invariant. Most importantly for practical purposes, the gauge invariance will remain true for any approximation within the transverse brackets $[\cdots]_{\mathrm{T}}$. The construction, in particular, shows that explicit final-state interactions occur here only in manifestly transverse contributions. This is similar to what was suggested by the approximation scheme of Ref. [9]. However, the present result shows that this property is generally true, independent of any approximation because of the $C^{\mu}$ subtraction in $[\cdots]_{\mathrm{T}}$. Implicit longitudinal FSI contributions are subsumed in $C_{\mathrm{L}}^{\mu}$ of Eq. (12) via its formal equivalence with Eq. (15) requiring the necessary condition (18).

Physical on-shell matrix elements,

$$
\mathcal{M}=\epsilon_{\mu} M^{\mu},
$$

are formed with transverse states $\epsilon_{\mu}$, either as the transverse photon polarization four-vector for real photons or, in the case of electroproduction, as the Dirac current of the

\footnotetext{
${ }^{2} \mathrm{~A}$ concise description of the construction procedure for the GIP current $C^{\mu}$ is given in the Appendix of Ref. [12]. In this context, note that the notation in Refs. [7,9,12] is slightly different from the one employed here. Note in particular that $C^{\mu}$ here is called $M_{\text {int }}^{\mu}$ in Ref. [12] Appendix, and that $C^{\mu}$ there is only the scalar coupling part of $C^{\mu}$ here.
}

electron between on-shell spinors times the photon propagator, $\epsilon^{\mu}=\bar{u}\left(p_{e}^{\prime}\right) \gamma^{\mu} u\left(p_{e}\right) / k^{2}$, where $k=p_{e}-p_{e}^{\prime}$ is the momentum difference transferred from the incoming and outgoing electron momenta to the photon. In both cases, the transversality condition $k_{\mu} \epsilon^{\mu}=0$ holds true and thus only transverse parts of the production current contribute for physical matrix elements,

$$
\begin{aligned}
\epsilon_{\mu} M^{\mu}= & \epsilon_{\mu}\left[M_{s}^{\mu}+M_{u}^{\mu}+M_{t}^{\mu}+M_{c}^{\mu}\right. \\
& \left.+X G_{0}\left(M_{u}^{\mu}+M_{t}^{\mu}+M_{c}^{\mu}\right)\right]_{\mathrm{T}},
\end{aligned}
$$

irrespective of whether the photon is real or virtual.

For the physical amplitude $\mathcal{M}$, therefore, the fact that the gWTI (8) was explicitly enabled via the tree-level contribution of Eq. (17) by adding and subtracting the transverse parts of the GIP current $C^{\mu}$ is quite irrelevant here since the current in the transverse brackets $[\cdots]_{\mathrm{T}}$ of $(20)$ is still the full production current $M^{\mu}$ stipulated to provide the $\mathrm{gWTI}$. The situation will change, however, if any of the mechanisms of $[\cdots]_{\mathrm{T}}$ in the exact equation (17) is approximated-which in practice generally will be unavoidable because of the complexity of the contributing mechanisms.

\section{A. Approximations}

By construction, any approximation of the transverse current $[\cdots]_{\mathrm{T}}$ in (17) will preserve full local gauge invariance. In general, however, such approximations will make the dependence on the GIP current $C^{\mu}$ manifest, in contrast to the exact amplitude (20).

The simplest approximation in (17) is to drop the entire $[\cdots]_{\mathrm{T}}$ current and replace the full current by the tree-level expression

$$
M^{\mu}=M_{s}^{\mu}+M_{u}^{\mu}+M_{t}^{\mu}+C^{\mu}
$$

depicted in Fig. 4. This approximation thus complies with the generic topological structure of a single-meson production current [15] and it provides the minimal structure necessary for producing the gWTI (8). Effectively, this corresponds to the replacement $M_{c}^{\mu}+X G_{0} B^{\mu} \rightarrow C^{\mu}$, with the contact-type GIP current $C^{\mu}$ providing here a phenomenological description for all exchange-current and FSI contributions depicted in Fig. 1. As the description in the Appendix of Ref. [12] shows, if necessary, $C^{\mu}$ will also account for a Kroll-Ruderman-type elementary contact current, as required, for example, for pseudovector pion-nucleon coupling in pion production. Moreover, additional phenomenological transverse contributions $T^{\mu}$ may be added without affecting gauge invariance, $C^{\mu} \rightarrow$ $C^{\mu}+T^{\mu}$, to better model the problem at hand [9]. Examples of applications of this tree-level approximation are given in Refs. [8,16-24].

A much more sophisticated approximation is to let $M_{c}^{\mu} \rightarrow C^{\mu}$, resulting in 


$$
\begin{aligned}
M^{\mu}= & M_{s}^{\mu}+M_{u}^{\mu}+M_{t}^{\mu}+C^{\mu} \\
& +\left[X G_{0}\left(M_{u}^{\mu}+M_{t}^{\mu}+C^{\mu}\right)\right]_{\mathrm{T}},
\end{aligned}
$$

where the dynamical details of the exchange-current contributions $U^{\mu} G_{0} F$ depicted in Fig. 1(b) are frozen out and summarily accounted for by the phenomenological current $C^{\mu}$. However, hadronic final-state interactions are fully incorporated. A variant of this approach was used for pion photoproduction in Ref. [11].

At a still more sophisticated level, one needs to explicitly consider the exchange currents $U^{\mu}$ contained in $M_{c}^{\mu}$ of Eq. (5), as depicted in Figs. 1(b) and (3). Structurally this results in

$$
\begin{aligned}
M^{\mu}= & M_{s}^{\mu}+M_{u}^{\mu}+M_{t}^{\mu}+C^{\mu} \\
& +\left[\tilde{M}_{c}^{\mu}-C^{\mu}+X G_{0}\left(M_{u}^{\mu}+M_{t}^{\mu}+\tilde{M}_{c}^{\mu}\right)\right]_{\mathrm{T}}
\end{aligned}
$$

where $\tilde{M}_{c}^{\mu}$ indicates the level of approximation of $M_{c}^{\mu}$. Details for expanding $M_{c}^{\mu}$ in terms of various exchange-current contributions are discussed in Ref. [9]. The subtraction of $C^{\mu}$ in the transverse part $[\cdots]_{\mathrm{T}}$ avoids double-counting because, as Eq. (22) shows, $C^{\mu}$ already provides a phenomenological account for the mechanisms contained in $\tilde{M}_{c}^{\mu}$. This necessary corrective term that follows from the exact equation (17) is not present in any of the previous approximate treatments of gauge invariance. Its importance is seen from the following considerations. The on-shell matrix element for this approximation, for both real and virtual photons, can be written simply as

$$
\begin{aligned}
\epsilon_{\mu} M^{\mu}= & \epsilon_{\mu}\left[M_{s}^{\mu}+M_{u}^{\mu}+M_{t}^{\mu}+\tilde{M}_{c}^{\mu}\right. \\
& \left.+X G_{0}\left(M_{u}^{\mu}+M_{t}^{\mu}+\tilde{M}_{c}^{\mu}\right)\right]_{\mathrm{T}},
\end{aligned}
$$

as can be seen from (20), and thus becomes independent of $C^{\mu}$. Without the subtraction, there would be an additional current $C^{\mu}$ in the transverse bracket $[\cdots]_{\mathrm{T}}$ of $(24)$ leading to an unwarranted double counting of contributions. Structurally, Eq. (24) is similar to Eq. (22) with $C^{\mu} \rightarrow \tilde{M}_{c}^{\mu}$. In other words, the focus is shifted from constructing a phenomenological GIP current $C^{\mu}$ to finding an acceptable approximation for the exchange current contributions contained in $M_{c}^{\mu}$. Even though $C^{\mu}$ does not appear explicitly in (24), this on-shell matrix element may still be considered fully compliant with local gauge invariance because of the underlying construction procedure in terms of (23). This is particularly relevant if $M^{\mu}$ in the form of Eq. (23) serves as (off-shell) input for other reactions like, for example, twomeson production processes [25], as explained in the subsequent discussion.

\section{DISCUSSION}

In summary, we have presented here an equivalent reformulation of the exact expressions for the production current $M^{\mu}$ that cleanly separates, in a model-independent manner, manifestly gauge-invariance-preserving tree-level expressions from transverse final-state interaction (FSI) terms, with microscopic reaction dynamics that are consistent for both real and virtual photons. Since the technically challenging parts of the dynamics-exchange currents and FSI in a coupled-channel framework-that generally require approximations appear here only in the transverse part, approximations will have no bearing on gauge invariance, for any level of sophistication of such approximations.

This separation of tree-level GIP terms and transverse remainder may look deceptively similar to what was suggested already in Ref. [9], however, there it was derived as an integral part of an elaborate approximation scheme. The present findings show now that this splitting is an exact consequence of the fact that local gauge invariance is equivalent to an implied (model-independent) longitudinal contact current $C_{\mathrm{L}}^{\mu}$ given by Eq. (15). Hence, any phenomenological GIP current $C^{\mu}$ that satisfies the necessary condition (18) will provide a manifestly gauge-invariant amplitude provided one subtracts its transverse part as in the $[\cdots]_{\mathrm{T}}$ bracket of Eq. (17) to make it model-independent and avoid double counting when considering approximations of $M_{c}^{\mu}$. This necessary subtraction is a new finding not present in previous approximate treatments. As a consequence, the dependence on a GIP current $C^{\mu}$ will only become manifest for approximations of the kind discussed in the context of Eqs. (21) and (22). For more sophisticated approximations like in Eq. (23) that explicitly take into account final-state interactions and exchange currents, the model-independent logitudinal part of $C^{\mu}$ is implied but it does not appear explicitly in the physical matrix element (24).

However, if one employs the single-meson production current $M^{\mu}$ as a contributing off-shell mechanism for twomeson production [25], one must necessarily use the (offshell) form (23) thus making the dependence on $C^{\mu}$ manifest as an internal current mechanism. This will ensure the overall local gauge invariance of electromagnetic twomeson production because, as shown in Ref. [25], the proof of the latter necessarily relies on the validity of the gWTI (8) for all contributing single-meson production currents.

More generally, off-shell single-meson production currents $M^{\mu}$ and their contributing mechanisms are required as input for many reactions. Examples including strangeness production and $N N$ bremsstrahlung processes are given in Refs. [26-30]. Rendering each one of these processes gauge invariant in a consistent manner necessarily requires that contributing single-meson production currents reproduce the gWTI (8) irrespective of the level of sophistication that goes into determining $M^{\mu}$ in practice.

Maintaining local gauge invariance is an important and indispensable ingredient for the consistent description of all electromagnetic processes. As we have shown here, doing 
so for the single-meson production current $M^{\mu}$ is a straightforward matter, in particular, when written in the form of Eq. (17) since the GIP part involves only the treelevel diagrams of Fig. 4. In practice, the real issue is finding useful approximations of all complex reaction mechanisms beyond the tree level to provide meaningful results with reasonable effort.

\section{ACKNOWLEDGMENTS}

The author gratefully acknowledges discussions with Kanzo Nakayama (UGA). The present work was supported in part by the U.S. Department of Energy, Office of Science, Office of Nuclear Physics, under Award No. DESC0016582.
[1] M.E. Peskin and D. V. Schroeder, An Introduction to Quantum Field Theory (Addison-Wesley, Reading, USA, 1995).

[2] E. Kazes, Generalized current conservation and low energy limit of photon interactions, Nuovo Cimento 13, 1226 (1959).

[3] S. D. Drell and T. D. Lee, Scaling properties and the boundstate nature of the physical nucleon, Phys. Rev. D 5, 1738 (1972).

[4] F. Gross and D. O. Riska, Current conservation and interaction currents in relativistic meson theories, Phys. Rev. C 36, 1928 (1987).

[5] K. Ohta, Electromagnetic interactions of extended nucleons, Phys. Rev. C 40, 1335 (1989).

[6] S. I. Nagorny and A. E. L. Dieperink, Gauge constraints and electromagnetic properties of off-shell particles, Eur. Phys. J. A 5, 417 (1999).

[7] H. Haberzettl, Gauge-invariant theory of pion photoproduction with dressed hadrons, Phys. Rev. C 56, 2041 (1997).

[8] H. Haberzettl, C. Bennhold, T. Mart, and T. Feuster, Gaugeinvariant tree-level photoproduction amplitudes with form factors, Phys. Rev. C 58, R40 (1998).

[9] H. Haberzettl, K. Nakayama, and S. Krewald, Gaugeinvariant approach to meson photoproduction including the final-state interaction, Phys. Rev. C 74, 045202 (2006).

[10] H. Haberzettl, F. Huang, and K. Nakayama, Dressing the electromagnetic nucleon current, Phys. Rev. C 83, 065502 (2011).

[11] F. Huang, M. Döring, H. Haberzettl, J. Haidenbauer, C. Hanhart, S. Krewald, U.-G. Meißner, and K. Nakayama, Pion photoproduction in a dynamical coupled-channels model, Phys. Rev. C 85, 054003 (2012).

[12] H. Haberzettl, X.-Y. Wang, and J. He, Preserving local gauge invariance with $t$-channel Regge exchange, Phys. Rev. C 92, 055503 (2015).

[13] J. C. Ward, An identity in quantum electrodynamics, Phys. Rev. 78, 182 (1950).

[14] Y. Takahashi, On the generalized Ward identity, Nuovo Cimento 6, 371 (1957).

[15] M. Gell-Mann and M. L. Goldberger, Scattering of lowenergy photons by particles of spin 1/2, Phys. Rev. 96, 1433 (1954).
[16] K. Nakayama and H. Haberzettl, Consistent analysis of the reactions $\gamma p \rightarrow p \eta^{\prime}$ and $p p \rightarrow p p \eta^{\prime}$, Phys. Rev. C 69, 065212 (2004).

[17] K. Nakayama and H. Haberzettl, Analyzing $\eta^{\prime}$ photoproduction data on the proton at energies of 1.5-2.3 GeV, Phys. Rev. C 73, 045211 (2006).

[18] Y. Oh, C. M. Ko, and K. Nakayama, Nucleon and $\Delta$ resonances in $K \Sigma(1385)$ photoproduction from nucleons, Phys. Rev. C 77, 045204 (2008).

[19] F. Huang, H. Haberzettl, and K. Nakayama, Combined analysis of $\eta^{\prime}$ production reactions: $\gamma N \rightarrow \eta^{\prime} N, N N \rightarrow N N \eta^{\prime}$, and $\pi N \rightarrow \eta^{\prime} N$, Phys. Rev. C 87, 054004 (2013).

[20] S.-H. Kim, A. Hosaka, and H.-C. Kim, Effects of $N(2000) 5 / 2^{+}, \quad N(2060) 5 / 2^{-}, \quad N(2120) 3 / 2^{-}, \quad$ and $N(2190) 7 / 2^{-}$on $K^{*} \Lambda$ photoproduction, Phys. Rev. D 90, 014021 (2014).

[21] X.-Y. Wang, J. He, and H. Haberzettl, Analysis of recent CLAS data on $\Sigma^{*}(1385)$ photoproduction off a neutron target, Phys. Rev. C 93, 045204 (2016).

[22] A. C. Wang, W. L. Wang, F. Huang, H. Haberzettl, and K. Nakayama, Nucleon resonances in $\gamma p \rightarrow K^{*+} \Lambda$, Phys. Rev. C 96, 035206 (2017).

[23] N. C. Wei, F. Huang, K. Nakayama, and D. M. Li, Nucleon resonances in $\gamma p \rightarrow \omega p$ reaction, Phys. Rev. D 100, 114026 (2019).

[24] Y. Zhang and F. Huang, Effects of $N(2000) 5 / 2^{+}$on $\gamma p \rightarrow K^{+} \Lambda(1405)$, Phys. Rev. C 103, 025207 (2021).

[25] H. Haberzettl, K. Nakayama, and Y. Oh, Theory of twomeson photo- and electroproduction off the nucleon, Phys. Rev. D 99, 053001 (2019).

[26] K. Nakayama, Y. Oh, and H. Haberzettl, Photoproduction of $\Xi$ off nucleons, Phys. Rev. C 74, 035205 (2006).

[27] K. Nakayama and H. Haberzettl, Interaction current in $p p \rightarrow p p \gamma$, Phys. Rev. C 80, 051001(R) (2009).

[28] K. Nakayama, Y. Oh, and H. Haberzettl, Combined analysis of $\eta$ meson hadro- and photoproduction off nucleons, J. Korean Phys. Soc. 59, 224 (2011).

[29] A. Johansson, H. Haberzettl, K. Nakayama, and C. Wilkin, Measurement and analysis of the $p p \rightarrow p p \gamma$ reaction at 310 MeV, Phys. Rev. C 83, 054001 (2011).

[30] H. Haberzettl and K. Nakayama, Gauge-invariant formulation of $N N \rightarrow N N \gamma$, Phys. Rev. C 85, 064001 (2012). 